\title{
Conceptual DFT Study of the Local Chemical Reactivity of the Colored BISARG Melanoidin and Its Protonated Derivative
}

\author{
Juan Frau ${ }^{1}$ and Daniel Glossman-Mitnik ${ }^{1,2 *}$ \\ ${ }^{1}$ Departament de Química, Universitat de les Illes Balears, Palma de Mallorca, Spain, ${ }^{2}$ Laboratorio Virtual NANOCOSMOS, \\ Centro de Investigación en Materiales Avanzados, Departamento de Medio Ambiente y Energía, Chihuahua, Mexico
}

OPEN ACCESS

Edited by:

Sam P. De Visser,

University of Manchester,

United Kingdom

Reviewed by:

Carles Curutchet,

Universitat de Barcelona, Spain

Mark Earl Casida

Université Grenoble Alpes, France

*Correspondence:

Daniel Glossman-Mitnik

daniel.glossman@cimav.edu.mx

Specialty section:

This article was submitted to

Theoretical and Computational

Chemistry,

a section of the journal

Frontiers in Chemistry

Received: 14 February 2018

Accepted: 09 April 2018

Published: 01 May 2018

Citation:

Frau J and Glossman-Mitnik D (2018) Conceptual DFT Study of the Local Chemical Reactivity of the Colored BISARG Melanoidin and Its

Protonated Derivative.

Front. Chem. 6:136.

doi: 10.3389/fchem.2018.00136
This computational study assessed eight fixed RSH (range-separated hybrid) density functionals that include CAM-B3LYP, LC- $\omega$ PBE, M11, MN12SX, N12SX, $\omega B$ 97, $\omega \mathrm{B} 97 \mathrm{X}$, and $\omega \mathrm{B} 97 \mathrm{XD}$ related to the Def2TZVP basis sets together with the SMD solvation model in the calculation the molecular structure and reactivity properties of the BISARG intermediate melanoidin pigment (5-(2-(E)-(Z)-5-[(2-furyl)methylidene]-3(4-acetylamino-4-carboxybutyl)-2-imino-1,3-dihydroimidazol-4-ylideneamino(E)-4-[(2furyl)methylidene]-5-oxo-1H-imidazol-1-yl)-2-acetylaminovaleric acid) and its protonated derivative, BISARG(p). The chemical reactivity descriptors for the systems were calculated via the Conceptual Density Functional Theory. The choice of active sites applicable to nucleophilic, electrophilic as well as radical attacks were made by linking them with Fukui functions indices, electrophilic and nucleophilic Parr functions, and the condensed Dual Descriptor $\Delta f(\mathbf{r})$. The study found the MN12SX and N12SX density functionals to be the most appropriate in predicting the chemical reactivity of the molecular systems under study starting from the knowledge of the HOMO, LUMO, and HOMO-LUMO gap energies.

Keywords: BISARG, conceptual DFT, chemical reactivity, dual descriptor, Parr functions

\section{INTRODUCTION}

Visual color in processed foods is largely due to colored products of Maillard or nonenzymic browning reactions. In spite of the longstanding aesthetic and practical interest in Maillard derived food coloring materials, relatively little is known about the chemical structures responsible for visual color (Rizzi, 1997). These chemical structures are known as Colored Maillard Reaction Products and can be isolated at intermediate stages during the melanoidin formation process.

Besides their interest as dye molecules which may be useful as food additives, but also as dyes for dye-sensitized solar cells (DSSC), these compounds have also antioxidant capabilities. Thus, they are amenable to be studied by analyzing their molecular reactivity properties.

One of these isolated molecules is named by the acronym BISARG and together with its protonated derivative, BISARG(p) have been experimentally studied as a part of a work related to the formation of melanidins (Hofmann, 1998) and we believe that it could be of interest to study their molecular reactivity by using the ideas of Conceptual DFT, in the same way of our previous works (Alvarado-González et al., 2013; Cervantes-Navarro and Glossman-Mitnik, 2013; Glossman-Mitnik, 2013a,b; Martínez-Araya et al., 2013a,b; SalgadoMorán et al., 2013; Glossman-Mitnik, 2014a,b,c,d; Martínez-Araya and Glossman-Mitnik, 2015; 
Martínez-Araya et al., 2015; Soto-Rojo et al., 2015; Frau et al., 2016a,b,c; Mendoza-Huízar et al., 2016; Frau et al., 2017a,b,c,d,e; Frau and Glossman-Mitnik, 2017a,b,c,d,e,f,g; Sastre et al., 2017).

The interest in using range-separated (RS) exchange correlation functionals in KS DFT is on the rise (Gledhill et al., 2016). The functionals tend to partition the $r_{12}^{-1}$ operator and exchange them into long- and short-range parts, whose range separation parameter $\omega$ controls the rate of attaining the long-range behavior. It is possible to fix the value of $\omega$. The value can also be nonempirically "tuned" through a system-by-system mechanism that minimizes some tuning norms. The basis of the optimal tuning approach is the knowledge that the energy that the HOMO should have, $\epsilon_{H}(\mathrm{~N})$, in exact KS as well as generalized $\mathrm{KS}$ theory for an $\mathrm{N}$ electron system, ought to be exactly $-\mathrm{IP}(\mathrm{N})$. Hence, IP represents the vertical ionization potential that is calculated by considering a particular functional energy difference $\mathrm{E}(\mathrm{N}-1)-\mathrm{E}(\mathrm{N})$. If approximate functionals are used, it is possible to have considerable differences between $\epsilon_{H}(\mathrm{~N})$, and $-\mathrm{IP}(\mathrm{N})$. Optimal tuning constitutes determining a system-specific range-separation parameter $\omega$ non-empirically in an RSE functional. Optionally, it also implied that several other parameters including $\epsilon_{H}(\mathrm{~N})=-\mathrm{IP}(\mathrm{N})$ are satisfied optimally (Jacquemin et al., 2014). Even though no equivalency exists to match this prescription of electron affinity (EA) coupled with LUMO in the case of neutral species, it is possible to say that $\epsilon_{H}(\mathrm{~N}+1)=-\mathrm{EA}(\mathrm{N})$, that is, the electron affinity of the neutral system is equal to minus the HOMO energy of the anion (SOMO), which facilitates the finding of an optimized value of $\omega$, and is then optimized to establish both properties simultaneously. Some concerns have been raised during the preparation of this paper regarding the validity of the ionization potential theorem (IP) within the context of Generalized Kohn-Sham (GKS) theory. However, it must stressed that Baer et al. (2010) and more recently Baerends et al. (2013) and Karolewski et al. (2013) have given arguments that the same criterion applies in GKS theories and with with hybrid and range-separated hybrid functionals. This will make it easy to predict the Conceptual DFT descriptors. In the past, the simultaneous prescription has been referred to as the "KID procedure" (for Koopmans in DFT), courtesy of the analogy it shares with the Koopmans' theorem within the Hartree-Fock theory. This SOMO energy will not be, in general, equal to the LUMO of the neutral, but if the difference between them, which we have called $\Delta \mathrm{SL}$, is small enough to be considered negligible for predictions of the Conceptual DFT descriptors, then the practical KID procedure will have a computational support.

This implies that the appropriateness of a particular density functional in making predictions of the Conceptual DFT descriptors directly by relying on the properties that the neutral molecule can be easily estimated. It only requires one to check the way that it has followed the KID procedure. Nevertheless, tuneoptimization depends on the system and must be performed for each molecule one at a time. Therefore, examining the various density functionals exhibiting significant accuracy across various types of databases in physics, chemistry, and where the $\omega$ value is fixed will determine how they perform the practical technique.
Thus, in this computational study we will assess eight density functionals in calculating the molecular properties and structure of the BISARG intermediate melanoidin pigment and its protonated derivative, BISARG(p). Following the same ideas of previous works, we will consider fixed RSH functional instead of the optimally-tuned RSH density functionals that have attained great success and have also supported the validity of the IP theorem in the context of the GKS theory (Stein et al., 2009a,b; Karolewski et al., 2011; Kuritz et al., 2011; Refaely-Abramson et al., 2011; Foster and Wong, 2012; Koppen et al., 2012; Kronik et al., 2012; Phillips et al., 2012a,b; Karolewski et al., 2013; Moore and Autschbach, 2013; Egger et al., 2014; Foster et al., 2014; Jacquemin et al., 2014; Niskanen and Hukka, 2014; Sun and Autschbach, 2014; Manna et al., 2015; Lima et al., 2016; Pereira et al., 2017).

\section{THEORETICAL BACKGROUND}

The theoretical background of this study is similar to the previous conducted research presented complete purposes, because this research is a component of a major project that it is in progress. If we consider the KID procedure mentioned in the Introduction together with a finite difference approximation, then the global reactivity descriptors can be written as:

\begin{tabular}{lll}
\hline Electronegativity & $\chi=-\frac{1}{2}(I+A) \approx \frac{1}{2}\left(\epsilon_{L}+\epsilon_{H}\right)$ & Parr and Yang (1984) \\
Global Hardness & $\eta=(I-A) \approx\left(\epsilon_{L}-\epsilon_{H}\right)$ & Parr and Yang (1984) \\
Electrophilicty & $\omega=\frac{\mu^{2}}{2 \eta}=\frac{(I+A)^{2}}{4(I-A)} \approx \frac{\left(\epsilon_{L}+\epsilon_{H}\right)^{2}}{4\left(\epsilon_{L}-\epsilon_{H}\right)}$ & Parr et al. (1999) \\
Electrodonating Power & $\omega^{-}=\frac{(3 /+A)^{2}}{16(I-A)} \approx \frac{\left(3 \epsilon_{H}+\epsilon_{L}\right)^{2}}{16 \eta}$ & Gázquez et al. (2007) \\
Electroaccepting Power & $\omega^{+}=\frac{(I+3 A)^{2}}{16(I-A)} \approx \frac{\left(\epsilon_{H}+3 \epsilon_{L}\right)^{2}}{16 \eta}$ & Gázquez et al. (2007) \\
Net electrophilicity & $\Delta \omega^{ \pm}=\omega^{+}-\left(-\omega^{-}\right)=\omega^{+}+\omega^{-}$ & Chattaraj et al. (2009)
\end{tabular}

where $\epsilon_{H}$ and $\epsilon_{L}$ are the energies of the highest occupied and the lowest unoccupied molecular orbitals (HOMO and LUMO), respectively.

Applying the same ideas, the definitions for the local reactivity descriptors are:

\begin{tabular}{lll}
\hline Nucleophilic Fukui Function & $f^{+}(\mathbf{r})=\rho_{N+1}(\mathbf{r})-\rho_{N}(\mathbf{r})$ & Parr and Yang (1984) \\
Electrophilic Fukui Function & $f^{-}(\mathbf{r})=\rho_{N}(\mathbf{r})-\rho_{N-1}(\mathbf{r})$ & Parr and Yang (1984) \\
Dual Descriptor & $\Delta f(\mathbf{r})=\left(\frac{\partial f(\mathbf{r})}{\partial N}\right)_{v(\mathbf{r})}$ & Morell et al. (2005, 2006) \\
Nucleophilic Parr Function & $P^{-}(\mathbf{r})=\rho_{S}^{r C}(\mathbf{r})$ & Domingo et al. (2013) \\
Electrophilic Parr Function & $P^{+}(\mathbf{r})=\rho_{S}^{r a}(\mathbf{r})$ & Domingo et al. (2013) \\
\hline
\end{tabular}

where $\rho_{N+1}(\mathbf{r}), \rho_{N}(\mathbf{r})$, and $\rho_{N-1}(\mathbf{r})$ are the electronic densities at point $\mathbf{r}$ for the system with $N+1, N$, and $N-1$ electrons, 
respectively, and $\rho_{s}^{r c}(\mathbf{r})$ and $\rho_{s}^{r a}(\mathbf{r})$ are related to the atomic spin density (ASD) at the $\mathbf{r}$ atom of the radical cation or anion of a given molecule, respectively (Domingo et al., 2016).

\section{SETTINGS AND COMPUTATIONAL METHODS}

Following the lines of our previous works, the computational studies were performed with the Gaussian 09 (Frisch et al., 2018) series of programs with density functional methods as implemented in the computational package. The basis set used in this work was Def2SVP for geometry optimization and frequencies, while Def2TZVP was considered for the calculation of the electronic properties (Weigend and Ahlrichs, 2005; Weigend, 2006). All the calculations were performed in the presence of water as the solvent by doing Integral Equation Formalism-Polarized Continuum Model (IEF-PCM) computations according to the SMD solvation model (Marenich et al., 2009).

For the calculation of the molecular structure and properties of the studied systems, we have chosen eight density functionals which are known to consistently provide satisfactory results for several structural and thermodynamic properties:

\begin{tabular}{|c|c|c|}
\hline CAM-B3LYP & $\begin{array}{l}\text { Long-range-corrected B3LYP by } \\
\text { the CAM method }\end{array}$ & Yanai et al. (2004) \\
\hline LC- $\omega$ PBE & $\begin{array}{l}\text { Long-range-corrected } \omega \mathrm{PBE} \\
\text { density functional }\end{array}$ & Henderson et al. (2009) \\
\hline M11 & $\begin{array}{l}\text { Range-separated hybrid } \\
\text { meta-GGA }\end{array}$ & Peverati and Truhlar (2011) \\
\hline MN12SX & $\begin{array}{l}\text { Range-separated hybrid } \\
\text { nonseparable meta-NGA }\end{array}$ & Peverati and Truhlar (2012) \\
\hline N12SX & Range-separated hybrid NGA & Peverati and Truhlar (2012) \\
\hline$\omega \mathrm{B} 97$ & $\begin{array}{l}\text { Long-range corrected density } \\
\text { functional }\end{array}$ & Chai and Head-Gordon (2008b) \\
\hline$\omega \mathrm{B} 97 \mathrm{X}$ & $\begin{array}{l}\text { Long-range corrected density } \\
\text { functional }\end{array}$ & Chai and Head-Gordon (2008b) \\
\hline$\omega \mathrm{B} 97 \mathrm{XD}$ & $\begin{array}{l}\omega \mathrm{B} 97 \mathrm{X} \text { version including } \\
\text { empirical dispersion }\end{array}$ & Chai and Head-Gordon (2008a) \\
\hline
\end{tabular}

In these functionals, GGA stands for generalized gradient approximation (in which the density functional depends on the up and down spin densities and their reduced gradient) and NGA stands for nonseparable gradient approximation (in which the density functional depends on the up/down spin densities and their reduced gradient, and also adopts a nonseparable form).

\section{RESULTS AND DISCUSSION}

The three-dimentional molecular structure of the BISARG system was built with the aid of molecular graphics program starting from structure presented in the original article (Hofmann, 1998). Starting from this, the molecular structure of its protonated derivative, BISARG(p) was built with the aid of a chemical visualization software. The pre-optimization of the systems was done using random sampling that involved molecular mechanics techniques and inclusion of the various torsional angles via the general MMFF94 force field (Halgren, 1996a,b,c, 1999; Halgren and Nachbar, 1996) through the Marvin View 17.15 program that constitutes an advanced chemical viewer suited to multiple and single chemical queries, structures and reactions (https://www.chemaxon.com). Afterwards, the structures that the resultant lower-energy conformers assumed for both molecules were reoptimized using the eight density functionals mentioned in the previous section together with the Def2SVP basis set as well as the SMD solvation model using water as the solvent.

The analysis of the results obtained in the study aimed at verifying that the KID procedure was fulfilled. On doing it previously, several descriptors associated with the results that HOMO and LUMO calculations obtained are related with results obtained using the vertical I and A following the $\triangle$ SCF procedure. A link exists between the three main descriptors and the simplest conformity to the Koopmans' theorem by linking $\epsilon_{H}$ with -I, $\epsilon_{L}$ with -A, and their behavior in describing the HOMOLUMO gap as $J_{I}=\left|\epsilon_{H}+E_{g s}(N-1)-E_{g s}(N)\right|, J_{A}=\mid \epsilon_{L}+E_{g s}(N)-$ $E_{g s}(N+1) \mid$, and $J_{H L}=\sqrt{J_{I}^{2}+J_{A}^{2}}$. Notably, the $J_{A}$ descriptor consists of an approximation that remains valid only when the HOMO that a radical anion has (the SOMO) shares similarity with the LUMO that the neutral system has. Consequently, we decided to design another descriptor $\triangle \mathrm{SL}$ (the difference between the SOMO and LUMO energies), to guide in verifying how the approximation is accurate.

The results of the calculation of the electronic energies of the neutral, positive and negative molecular systems (in au) of BISARG and BISARG(p), the HOMO, LUMO, and SOMO orbital energies (in $\mathrm{eV}$ ), $J_{I}, J_{A}, J_{H L}$, and $\Delta \mathrm{SL}$ descriptors calculated with the eight density functionals and the Def2TZVP basis set using water as solvent simulated with the SMD parametrization of the IEF-PCM model are presented in Tables 1, 2.

As presented in previous works, we considered four other descriptors that analyze how well the studied density functionals are useful for the prediction of the electronegativity $\chi$, the global hardness $\eta$, and the global electrophilicity $\omega$, and for a combination of these Conceptual DFT descriptors, considering only the energies of the HOMO and LUMO or the vertical I and A: $J_{\chi}=\left|\chi-\chi_{K}\right|, J_{\eta}=\left|\eta-\eta_{K}\right|, J_{\omega}=\left|\omega-\omega_{K}\right|$, and $J_{C D F T}=\sqrt{J_{\chi}^{2}+J_{\eta}^{2}+J_{\omega}^{2}}$, where CDFT stands for Conceptual DFT. The underscript $\mathrm{K}$ stands for the descriptor calculated by applying the KID procedure.The results of the calculations of $J_{\chi}, J_{\eta}, J_{\omega}$, and $J_{C D F T}$ for the low-energy conformers of BISARG and BISARG(p) in water are displayed in Tables 3, 4, respectively.

As Tables 1-4 provide, the KID procedure applies accurately from MN12SX and N12SX density functionals that are rangeseparated hybrid meta-NGA as well as range-separated hybrid NGA density functionals respectively. In fact, the values of $J_{I}, J_{A}$, and $J_{H L}$ are actually not zero. Nevertheless, the results tend to be impressive especially for the MN12SX density functional. As well, the $\Delta$ SL descriptor reaches the minimum values when MN12SX and N12SX density functionals are used in the calculations. This implies that there are sufficient justifications to assume that the 
TABLE 1 | Electronic energies of the neutral, positive and negative molecular systems (in au) of the BISARG molecule, the HOMO, LUMO, and SOMO orbital energies (in $\mathrm{eV}$ ), $J_{l}, J_{A}, J_{H L}$, and $\triangle S L$ descriptors (also in eV) calculated with the eight RSH density functionals and the Def2TZVP basis set using water as solvent simulated with the SMD parametrization of the IEF-PCM model.

\begin{tabular}{lcccccccccc}
\hline & Eo & E+ & E- & HOMO & LUMO & SOMO & $\boldsymbol{J}_{\boldsymbol{I}}$ & $\boldsymbol{J}_{\boldsymbol{A}}$ & $\boldsymbol{J}_{\boldsymbol{H L}}$ & $\boldsymbol{\Delta S L}$ \\
\hline CAM-B3LYP & -2276.0764 & -2275.8795 & -2276.1832 & -6.6124 & -1.6898 & -4.1310 & 1.2522 & 1.2168 & 1.7458 & 2.4412 \\
LC- $\omega$ BPE & -2275.6818 & -2275.4754 & -2275.7958 & -7.8736 & -0.9566 & -5.2792 & 2.2572 & 2.1454 & 3.1139 & 4.3226 \\
M11 & -2275.9123 & -2275.7056 & -2276.0238 & -7.6575 & -1.1204 & -4.9627 & 2.0321 & 1.9146 & 2.7920 & 3.8422 \\
MN12SX & -2275.1376 & -2274.9395 & -2275.2504 & -5.3883 & -3.0639 & -3.0873 & 0.0022 & 0.0082 & 0.0084 & 0.0144 \\
N12SX & -2276.0990 & -2275.9088 & -2276.2081 & -5.1959 & -2.9278 & -3.0064 & 0.0218 & 0.0433 & 0.0484 & 0.0787 \\
$\omega$ B97 & -2276.7888 & -2276.5871 & -2276.8951 & -7.7315 & -0.7982 & -5.0476 & 2.2420 & 2.0947 & 3.0685 & 4.2494 \\
$\omega$ B97X & -2276.5890 & -2276.3879 & -2276.6957 & -7.5543 & -0.9354 & -4.9069 & 2.0819 & 1.9682 & 2.8652 & 3.9715 \\
$\omega$ B97XD & -2276.4388 & -2276.2394 & -2276.5463 & -7.2139 & -1.1972 & -4.6660 & 1.7858 & 1.7271 & 2.4842 & 3.4688 \\
\hline
\end{tabular}

TABLE 2 | Electronic energies of the neutral, positive and negative molecular systems (in au) of the protonated BISARG(p) molecule, the HOMO, LUMO, and SOMO orbital energies (in eV), $J_{l}, J_{A}, J_{H L}$, and $\triangle S L$ descriptors (also in eV) calculated with the eight RSH density functionals and the Def2TZVP basis set using water as solvent simulated with the SMD parametrization of the IEF-PCM model.

\begin{tabular}{|c|c|c|c|c|c|c|c|c|c|c|}
\hline & Eo & $\mathrm{E}+$ & E- & НОМо & LUMO & SOMO & $J_{I}$ & $J_{A}$ & $J_{H L}$ & $\Delta \mathrm{SL}$ \\
\hline CAM-B3LYP & -2276.5424 & -2276.3458 & -2276.6659 & -6.6364 & -2.0844 & -4.6328 & 1.2862 & 1.2775 & 1.8128 & 2.5484 \\
\hline LC- $\omega$ BPE & -2276.1485 & -2275.9438 & -2276.2828 & -7.8698 & -1.3822 & -5.8926 & 2.2969 & 2.2703 & 3.2296 & 4.5103 \\
\hline M11 & -2276.3780 & -2276.1724 & -2276.5077 & -7.6670 & -1.5308 & -5.5304 & 2.0719 & 2.0011 & 2.8804 & 3.9995 \\
\hline MN12SX & -2275.6003 & -2275.4000 & -2275.7267 & -5.4495 & -3.4299 & -3.4481 & 0.0014 & 0.0114 & 0.0114 & 0.0182 \\
\hline N12SX & -2276.5694 & -2276.3768 & -2276.6925 & -5.2653 & -3.2944 & -3.3910 & 0.0237 & 0.0558 & 0.0604 & 0.0966 \\
\hline$\omega \mathrm{B} 97$ & -2277.2550 & -2277.0549 & -2277.3813 & -7.7220 & -1.2241 & -5.6655 & 2.2784 & 2.2150 & 3.1776 & 4.4314 \\
\hline$\omega \mathrm{B} 97 \mathrm{X}$ & -2277.0557 & -2276.8559 & -2277.1814 & -7.5546 & -1.3488 & -5.4980 & 2.1179 & 2.0727 & 2.9634 & 4.1492 \\
\hline$\omega \mathrm{B97XD}$ & -2276.9093 & -2276.7104 & -2277.0339 & -7.2329 & -1.5926 & -5.1894 & 1.8210 & 1.7976 & 2.5588 & 3.5967 \\
\hline
\end{tabular}

TABLE $3 \mid J_{\chi}, J_{\eta}, J_{\omega}$, and $J_{C D F T}$ (in eV) of the BISARG intermediate melanoidin pigment.

\begin{tabular}{lcccc}
\hline & $\boldsymbol{J}_{\boldsymbol{\chi}}$ & $\boldsymbol{J}_{\boldsymbol{\eta}}$ & $\boldsymbol{J}_{\boldsymbol{\omega}}$ & $\boldsymbol{J}_{\boldsymbol{C D F T}}$ \\
\hline CAM-B3LYP & 0.0185 & 2.4706 & 1.7322 & 3.0174 \\
LC- $\omega$ BPE & 0.0552 & 4.4023 & 2.3704 & 5.0002 \\
M11 & 0.0591 & 3.9462 & 2.1447 & 4.4917 \\
MN12SX & 0.0045 & 0.0030 & 0.0131 & 0.0142 \\
N12SX & 0.0109 & 0.0610 & 0.1205 & 0.1355 \\
$\omega$ B97 & 0.0738 & 4.3370 & 2.0710 & 4.8067 \\
$\omega$ B97X & 0.0565 & 4.0499 & 2.0530 & 4.5409 \\
$\omega$ B97XD & 0.0294 & 3.5156 & 2.0168 & 4.0531 \\
\hline
\end{tabular}

LUMO of the neutral approximates the electron affinity. The same density functionals follow the KID procedure in the rest of the descriptors such as $J_{\chi}, J_{\eta}, J_{\omega}$, and $J_{C D F T}$.

Having verified that the MN12SX/Def2TZVP model chemistry is a good choice for the calculation of the global reactivity descriptors, we now present the optimized molecular structures of BISARG and BISARG(p) in water in Supplementary Figures 1, 2. Meanwhile, the calculated bond lengths and bond angles for both cases are shown in Supplementary Tables 1-4.

As a summary of the previous results, the global reactivity descriptors for the BISARG and BISARG(p) molecules calculated
TABLE $4 \mid J_{\chi}, J_{\eta}, J_{\omega}$, and $J_{C D F T}$ (in eV) of the protonated BISARG(p) intermediate melanoidin pigment.

\begin{tabular}{lcccc}
\hline & $\boldsymbol{J}_{\chi}$ & $\boldsymbol{J}_{\boldsymbol{\eta}}$ & $\boldsymbol{J}_{\boldsymbol{\omega}}$ & $\boldsymbol{J}_{\boldsymbol{C D F T}}$ \\
\hline CAM-B3LYP & 0.0046 & 2.5626 & 2.6800 & 3.7080 \\
LC- $\omega$ BPE & 0.0131 & 4.5716 & 3.9039 & 6.0117 \\
M11 & 0.0363 & 4.0705 & 3.3156 & 5.2501 \\
MN12SX & 0.0059 & 0.0084 & 0.0334 & 0.0349 \\
N12SX & 0.0161 & 0.0795 & 0.2316 & 0.2454 \\
$\omega$ B97 & 0.0316 & 4.4894 & 3.3713 & 5.6144 \\
$\omega$ B97X & 0.0225 & 4.1892 & 3.2674 & 5.3128 \\
$\omega$ B97XD & 0.0107 & 3.6182 & 3.0654 & 4.7422 \\
\hline
\end{tabular}

with the MN12SX/Def2TZVP model chemistry in water are presented in Table 5.

The calculations of the condensed Fukui functions and dual descriptor are done by using the Chemcraft molecular analysis program to extract the Mulliken and NPA atomic charges (Zhurko and Zhurko, 2012) beginning with single-point energy calculations involving the MN12SX density functional that uses the Def2TZVP basis set in line with the SMD solvation model, and water utilized as the solvent.

Considering the potential application the studied molecules as antioxidants, it is of interest to get insight into the active sites for radical attack. Graphical representations of the radical Fukui 
TABLE 5 | Global reactivity descriptors for the BISARG intermediate melanoidin pigment and its protonated derivative BISARGd(p) calculated with the MN12SX density functional.

Electronegativity $(\chi)$ Chemical hardness $(\eta)$ Electrophilicity $(\omega)$

\begin{tabular}{lccc}
\hline BISARG & 4.2253 & 2.3240 & 3.8410 \\
BISARG $(p)$ & 4.4389 & 2.0193 & 4.8790 \\
\hline & $\begin{array}{c}\text { Electrodonating } \\
\text { Power }\left(\omega^{-}\right)\end{array}$ & $\begin{array}{c}\text { Electroaccepting } \\
\text { Power }\left(\boldsymbol{\omega}^{+}\right)\end{array}$ & $\begin{array}{c}\text { Net electrophilicity } \\
\left(\boldsymbol{\Delta} \boldsymbol{\omega}^{ \pm}\right)\end{array}$ \\
\hline BISARG & 5.8449 & 4.4318 & 10.2767 \\
BISARG $(p)$ & 7.1172 & 5.6637 & 12.7809
\end{tabular}

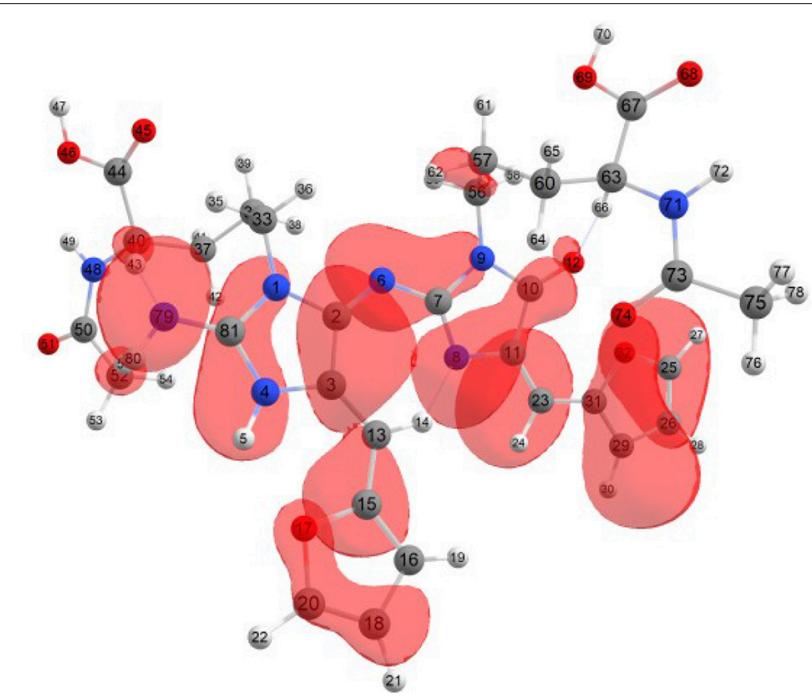

FIGURE 1 | A graphical schematic representation of the radical Fukui function $f^{0}$ over the atomic sites of the BISARG intermediate melanoidin pigment.

function $f^{0}$ calculated with the MN12SX/Def2TZVP model chemistry for both systems in water are presented in Figures 1, 2.

The condensed electrophilic and nucleophilic Parr functions $P_{k}^{+}$and $P_{k}^{-}$over the atoms of the BISARG and BISARG(p) molecules in water have been calculated by extracting the Mulliken and Hirshfeld (or CM5) atomic charges using the Chemcraft molecular analysis program (Zhurko and Zhurko, 2012) starting from single-point energy calculations of the ionic species with the MN12SX density functional using the Def2TZVP basis set in the presence of the solvents according to the SMD solvation model.

The results for the condensed dual descriptor calculated with Mulliken atomic charges $\Delta \mathrm{f}_{k}(\mathrm{M})$, with NPA atomic charges $\Delta \mathrm{f}_{k}(\mathrm{~N})$, the electrophilic and nucleophilic Parr functions with Mulliken atomic spin densities $P_{k}^{+}(\mathrm{M})$ and $P_{k}^{-}(\mathrm{M})$, and the electrophilic and nucleophilic Parr functions with Hirshfeld (or CM5) atomic spin densities $P_{k}^{+}(\mathrm{H})$ and $P_{k}^{-}(\mathrm{H})$ are displayed in Tables 6, 7 for the BISARG and BISARG(p) molecules in water, respectively, while Figures 3, 4 show schematic representations of the molecules with the numbering of the most important reactive sites according to the results in Tables 6, 7 .

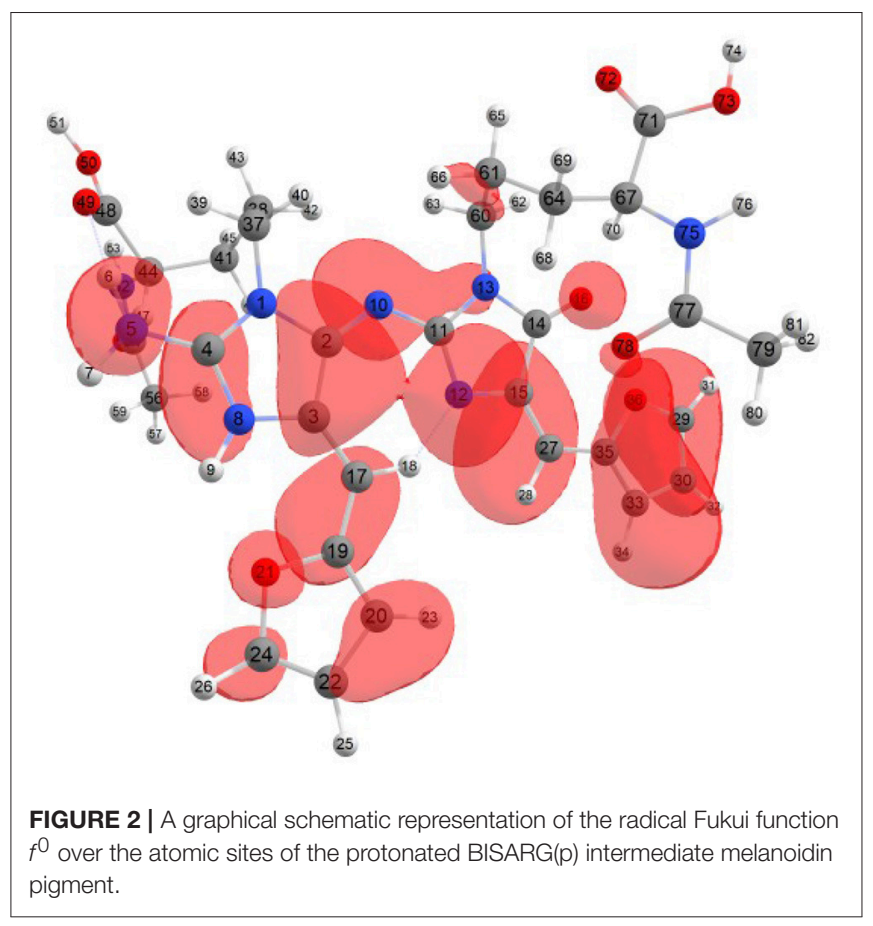

TABLE 6 | The condensed dual descriptor calculated with Mulliken atomic charges $\Delta f_{k}(M)$, and with NPA atomic charges $\Delta f_{k}(N)$, the electrophilic and nucleophilic Parr functions with Mulliken atomic spin densities $P_{k}^{+}(\mathrm{M})$ and $P_{k}^{-}(\mathrm{M})$, and the electrophilic and nucleophilic Parr functions with Hirshfeld (or CM5) atomic spin densities $P_{k}^{+}(\mathrm{H})$ and $P_{k}^{-}(\mathrm{H})$ for the BISARG melanoidin molecule. Hydrogens and atomic sites where the absolute value of the dual descriptor is lower than 1 are not shown.

\begin{tabular}{lrrrrrr}
\hline Atom & $\boldsymbol{\Delta f}_{\boldsymbol{k}} \mathbf{( M )}$ & $\Delta \mathbf{f}_{\boldsymbol{k}}(\mathbf{N})$ & $\boldsymbol{P}_{\boldsymbol{k}}^{+}(\mathbf{M})$ & $\boldsymbol{P}_{\boldsymbol{k}}^{-}(\mathbf{M})$ & $\boldsymbol{P}_{\boldsymbol{k}}^{+}(\mathbf{H})$ & $\boldsymbol{P}_{\boldsymbol{k}}^{-}(\mathbf{H})$ \\
\hline 1N & 1.95 & 0.17 & 0.0265 & 0.0052 & 0.0355 & 0.0013 \\
2C & 12.26 & 9.75 & 0.2046 & -0.0477 & 0.1282 & -0.0128 \\
3C & -2.86 & -3.50 & -0.0495 & 0.0454 & 0.0107 & 0.0382 \\
4N & -4.84 & -3.07 & -0.0093 & 0.0664 & -0.0078 & 0.0502 \\
6N & -4.90 & -4.93 & -0.0386 & 0.1255 & -0.0014 & 0.0842 \\
7C & 4.31 & 3.52 & 0.0990 & -0.0287 & 0.0724 & 0.0118 \\
8N & -7.69 & -5.30 & 0.0121 & 0.1827 & 0.0144 & 0.1373 \\
10C & 3.75 & 3.52 & 0.0606 & -0.0093 & 0.0446 & 0.0055 \\
11C & -4.19 & -3.69 & -0.0307 & 0.0601 & 0.0164 & 0.0672 \\
13C & 3.64 & 3.40 & 0.1828 & 0.0879 & 0.1057 & 0.0693 \\
15C & -3.10 & -0.39 & -0.0496 & 0.0160 & -0.0010 & 0.0274 \\
18C & -1.20 & -0.94 & -0.0285 & -0.0154 & -0.0028 & 0.0082 \\
20C & -2.43 & -1.71 & 0.0724 & 0.1095 & 0.0502 & 0.0801 \\
23C & 8.06 & 7.25 & 0.2653 & 0.0842 & 0.1563 & 0.0720 \\
25C & -3.21 & -1.99 & 0.1020 & 0.1482 & 0.0709 & 0.1094 \\
26C & -1.89 & -1.47 & -0.0388 & -0.0136 & -0.0033 & 0.0150 \\
31C & -4.63 & -0.94 & -0.0663 & 0.0355 & 0.0001 & 0.0422 \\
320 & 1.16 & 1.07 & 0.0184 & -0.0118 & 0.0194 & 0.0009 \\
79N & 2.58 & 1.75 & 0.0473 & -0.0153 & 0.0355 & -0.0113 \\
81C & 1.28 & 0.19 & 0.0000 & -0.0043 & 0.0127 & 0.0010 \\
\hline & & & & & &
\end{tabular}

From the results for the local reactivity descriptors in Table 6, it can be concluded that $\mathrm{C} 2$ and $\mathrm{C} 23$ will be the preferred sites for a nucleophilic attack and that these atoms will act as electrophilic 
TABLE 7 | The condensed dual descriptor calculated with Mulliken atomic charges $\Delta \mathrm{f}_{k}(\mathrm{M})$, and with NPA atomic charges $\Delta \mathrm{f}_{k}(\mathrm{~N})$, the electrophilic and nucleophilic Parr functions with Mulliken atomic spin densities $P_{k}^{+}(\mathrm{M})$ and $P_{k}^{-}(\mathrm{M})$, and the electrophilic and nucleophilic Parr functions with Hirshfeld (or CM5) atomic spin densities $P_{k}^{+}(\mathrm{H})$ and $P_{k}^{-}(\mathrm{H})$ for the BISARG(p) melanoidin molecule. Hydrogens and atomic sites where the absolute value of the dual descriptor is lower than 1 are not shown.

\begin{tabular}{|c|c|c|c|c|c|c|}
\hline Atom & $\Delta \mathrm{f}_{k}(\mathrm{M})$ & $\Delta \mathbf{f}_{\boldsymbol{k}}(\mathbf{N})$ & $P_{k}^{+}(\mathrm{M})$ & $P_{k}^{-}(\mathrm{M})$ & $P_{k}^{+}(\mathrm{H})$ & $P_{k}^{-}(\mathrm{H})$ \\
\hline $1 \mathrm{~N}$ & -0.28 & -1.09 & -0.0083 & 0.0179 & 0.0158 & 0.0112 \\
\hline $2 \mathrm{C}$ & 12.50 & 8.97 & 0.2317 & -0.0230 & 0.1412 & -0.0021 \\
\hline $4 C$ & 3.40 & 2.52 & 0.0402 & -0.0084 & 0.0341 & -0.0032 \\
\hline $5 \mathrm{~N}$ & 2.40 & 2.14 & 0.0260 & -0.0076 & 0.0234 & -0.0062 \\
\hline $8 \mathrm{~N}$ & -1.30 & -0.84 & -0.0074 & 0.0218 & -0.0056 & 0.0161 \\
\hline $12 \mathrm{~N}$ & -6.95 & -3.44 & 0.0425 & 0.1817 & 0.0308 & 0.1445 \\
\hline $15 \mathrm{C}$ & -10.14 & -7.30 & -0.0588 & 0.1222 & -0.0089 & 0.1049 \\
\hline 160 & -3.36 & -4.03 & 0.0136 & 0.0797 & 0.0125 & 0.0662 \\
\hline $17 \mathrm{C}$ & 13.43 & 8.74 & 0.3116 & 0.0363 & 0.1795 & 0.0297 \\
\hline $19 \mathrm{C}$ & -1.32 & 1.08 & -0.0883 & 0.0093 & -0.0042 & 0.0134 \\
\hline $20 \mathrm{C}$ & 6.25 & 4.80 & 0.1683 & 0.0409 & 0.1008 & 0.0299 \\
\hline 210 & 1.44 & 1.41 & 0.0184 & -0.0043 & 0.0200 & 0.0002 \\
\hline $24 C$ & 3.85 & 2.95 & 0.1294 & 0.0546 & 0.0900 & 0.0401 \\
\hline $27 \mathrm{C}$ & 2.39 & 4.54 & 0.1996 & 0.0718 & 0.1140 & 0.0759 \\
\hline $29 \mathrm{C}$ & -7.81 & -5.59 & 0.0807 & 0.1977 & 0.0566 & 0.1467 \\
\hline $30 \mathrm{C}$ & -2.76 & -2.17 & -0.0284 & -0.0127 & -0.0017 & 0.0222 \\
\hline $33 \mathrm{C}$ & -3.67 & -2.45 & 0.1007 & 0.1274 & 0.0610 & 0.0975 \\
\hline $35 \mathrm{C}$ & -7.01 & -2.75 & -0.0547 & 0.0663 & -0.0039 & 0.0638 \\
\hline
\end{tabular}

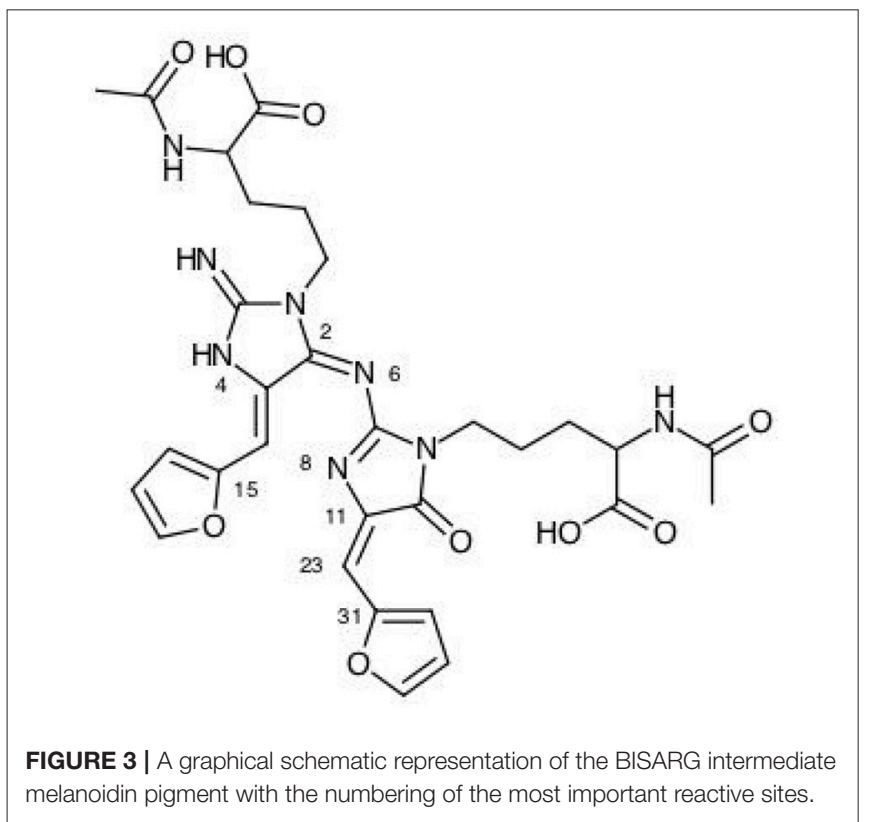

species in a chemical reaction. In turn, it can be appreciated that $\mathrm{N} 8$ will be prone to electrophilic attacks and that this atomic site will act as a nucleophilic species in chemical reactions that involve the BISARG molecule in water. In turn, for the case of the BISARG(p) molecule in water, C2 and C17 will be the preferred

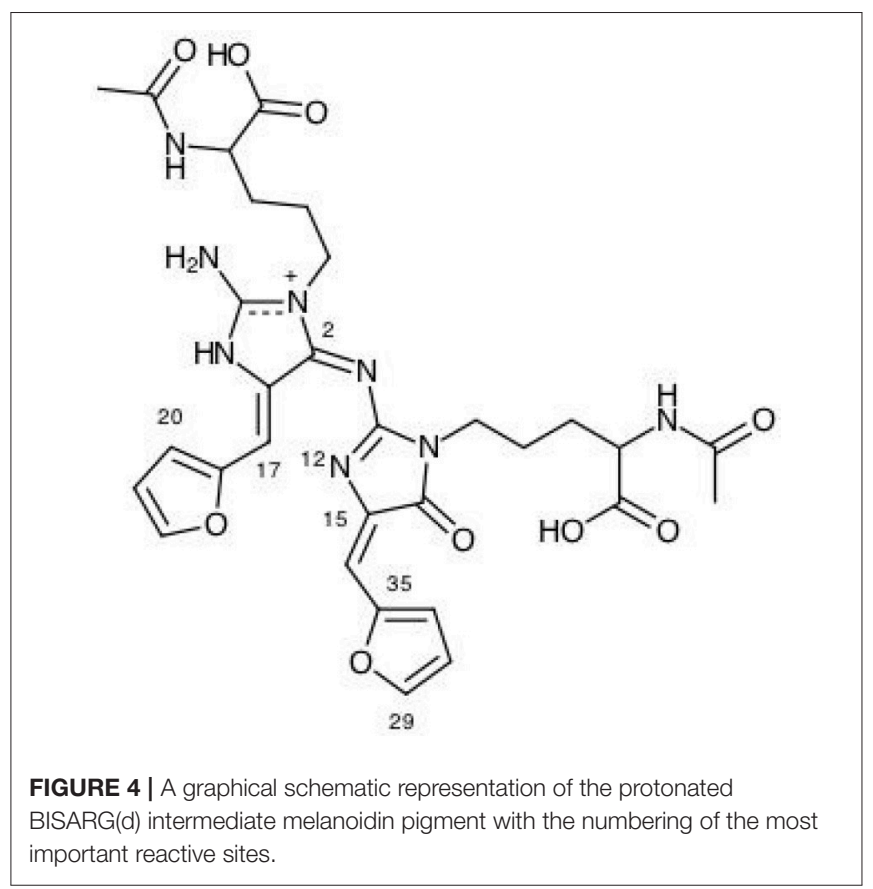

sites for a nucleophilic attack while C15 and C29 will be the sites for electrophilic reactions.

\section{CONCLUSIONS}

Eight fixed RSH density functionals, including CAM-B3LYP, LC$\omega \mathrm{PBE}, \mathrm{M} 11, \mathrm{MN} 12 \mathrm{SX}, \mathrm{N} 12 \mathrm{SX}, \omega \mathrm{B} 97, \omega \mathrm{B} 97 \mathrm{X}$, and $\omega \mathrm{B} 97 \mathrm{XD}$, were examined to determine whether they fulfill the empirical KID procedure so as to provide computational support for this common practice. The assessment was conducted by comparing the values from HOMO and LUMO calculations to those generated by the $\triangle \mathrm{SCF}$ technique for the BISARG molecule and its protonated derivative, BISARG(p). BISARG and BISARG(p) are intermediate melanoidin pigments that are of academic and industrial interest. The study has observed that the rangeseparated and hybrid meta-NGA density functionals tend to be the most suited in meeting this goal. Thus, they can be suitable alternatives to density functionals where the behavior of them is optimally tuned using a gap-fitting procedure. They also exhibit the desirable prospect of benefiting future studies aimed at understanding the chemical reactivity of colored melanoidins with larger molecular weights when reducing sugars react with proteins and peptides.

It is not the goal of Computational Chemistry to perform studies to reproduce known experimental results except in the case that they can be used for the calibration of a particular technique. Instead, it can be useful to predict in advance the structural and chemical reactivity characteristics of new or unknown molecular systems whose properties have not been reported and as guide for future research. As far as we know, there are no reports in the literature about the chemical reactivity properties for the molecular systems considered in this work and it is not possible to perform any kind of comparisons. 
However, the present study shows that with an adequate choice of the model chemistry we have been able to predict the sites of interaction of the BISARG and BISARG(p) molecules with impressive accuracy starting from the knowledge of the HOMO, LUMO, and HOMO-LUMO gap energies of the studied systems. This involves having DFT-based reactivity descriptors, including Fukui functions, Parr functions, and Dual Descriptor calculations. In conclusion, the Conceptual DFT descriptors are useful in characterizing and describing the preferred reactive sites and in comprehensively explaining the reactivity of the molecules.

\section{AUTHOR CONTRIBUTIONS}

DG-M conceived and designed the research and headed, wrote and revised the manuscript, while JF contributed to the writing and the revision of the article.

\section{REFERENCES}

Alvarado-González, M., Flores-Holguín, N., and Glossman-Mitnik, D. (2013). Computational nanochemistry study of the molecular structure and properties of the chlorophyll a molecule. Int. J. Photoener. 2013:8. doi: $10.1155 / 2013 / 424620$

Baer, R., Livshits, E., and Salzner, U. (2010). Tuned range-separated hybrids in density functional theory. Annu. Rev. Phys. Chem. 61, 85-109. doi: 10.1146/annurev.physchem.012809.103321

Baerends, E. J., Gritsenko, O. V., and van Meer, R. (2013). The Kohn-Sham gap, the fundamental gap and the optical gap: the physical meaning of occupied and virtual Kohn-Sham orbital energies. Phys. Chem. Chem. Phys. 15, 16408-16425. doi: $10.1039 / \mathrm{c} 3 \mathrm{cp} 52547 \mathrm{c}$

Cervantes-Navarro, F., and Glossman-Mitnik, D. (2013). Density functional study of the effects of substituents on the chemical reactivity of the Indigo molecule. J. Theory Comput. Chem. 12:1350013. doi: 10.1142/S0219633613500132

Chai, J., and Head-Gordon, M. (2008a). Long-range corrected hybrid density functionals with damped atom-atom dispersion corrections. Phys. Chem. Chem. Phys. 10, 6615-6620. doi: 10.1039/b810189b

Chai, J., and Head-Gordon, M. (2008b). Systematic optimization of longrange corrected hybrid density functionals. J. Chem. Phys. 128:084106. doi: 10.1063/1.2834918

Chattaraj, P., Chakraborty, A., and Giri, S. (2009). Net electrophilicity. J. Phys. Chem. A 113, 10068-10074. doi: 10.1021/jp904674x

Domingo, L. R., Pérez, P., and Sáez, J. (2013). Understanding the local reactivity in polar organic reactions through electrophilic and nucleophilic parr functions. RSC Adv. 3, 1486-1494. doi: 10.1039/C2RA22886F

Domingo, L. R., Ríos-Gutiérrez, M., and Pérez, P. (2016). Applications of the conceptual density functional theory indices to organic chemistry reactivity. Molecules 21:e748. doi: 10.3390/molecules21060748

Egger, D. A., Weissman, S., Refaely-Abramson, S., Sharifzadeh, S., Dauth, M., Baer, R., et al. (2014). Outer-valence electron spectra of prototypical aromatic heterocycles from an optimally tuned range-separated hybrid functional. J. Chem. Theory Comput. 10, 1934-1952. doi: 10.1021/ct400956h

Foster, M. E., Azoulay, J. D., Wong, B. M., and Allendorf, M. D. (2014). Novel metal-organic framework linkers for light harvesting applications. Chem. Sci. 5, 2081-2090. doi: 10.1021/jp0622381

Foster, M. E., and Wong, B. M. (2012). Nonempirically tuned rangeseparated DFT accurately predicts both fundamental and excitation gaps in DNA and RNA nucleobases. J. Chem. Theory Comput. 8, 2682-2687. doi: $10.1021 / \mathrm{ct} 300420 \mathrm{f}$

Frau, J., and Glossman-Mitnik, D. (2017a). A comparative study of the glycating power of simple carbohydrates in the maillard reaction by means of conceptual DFT descriptors. Br. J. Appl. Sci. Technol. 21:32795. doi: 10.9734/BJAST/2017/32795

\section{ACKNOWLEDGMENTS}

This work has been partially supported by CIMAV, SC, and Consejo Nacional de Ciencia y Tecnología (CONACYT, Mexico) through Grant 219566-2014 for Basic Science Research. DG$\mathrm{M}$ conducted this work while a Visiting Lecturer at the University of the Balearic Islands from which support is gratefully acknowledged. This work was cofunded by the Ministerio de Economía y Competitividad (MINECO) and the European Fund for Regional Development (FEDER) (CTQ2014-55835-R).

\section{SUPPLEMENTARY MATERIAL}

The Supplementary Material for this article can be found online at: https://www.frontiersin.org/articles/10.3389/fchem. 2018.00136/full\#supplementary-material

Frau, J., and Glossman-Mitnik, D. (2017b). A conceptual DFT study of the molecular properties of glycating carbonyl compounds. Chem. Cent. J. 11:8. doi: 10.1186/s13065-017-0239-7

Frau, J., and Glossman-Mitnik, D. (2017c). Chemical reactivity theory study of advanced glycation endproduct inhibitors. Molecules 22:226. doi: 10.3390/molecules22020226

Frau, J., and Glossman-Mitnik, D. (2017d). Computational prediction of the reactivity sites of Alzheimer amyloid $\beta$-peptides $\mathrm{A} \beta 40$ and $\mathrm{A} \beta 42$. ChemXpress 10:120.

Frau, J., and Glossman-Mitnik, D. (2017e). Conceptual DFT descriptors of amino acids with potential corrosion inhibition properties calculated with the latest Minnesota density functionals. Front. Chem. 5:16. doi: $10.3389 /$ fchem.2017.00016

Frau, J., and Glossman-Mitnik, D. (2017f). Molecular modeling study of the structures, properties and glycating power of some reducing disacharides. MOJ Drug Design Dev. Ther. 1:00003. doi: 10.15406/mojddt.2017.01.0003

Frau, J., and Glossman-Mitnik, D. (2017g). Pyridoxamine derivatives as non enzymatic glycation inhibitors: the conceptual DFT viewpoint. Res. J. Life Sci. Bioinform. Pharm. Chem. Sci. 2, 103-122. doi: 10.26479/2017.0206.09

Frau, J., Hernández-Haro, N., and Glossman-Mitnik, D. (2017a). Computational prediction of the pKas of small peptides through conceptual DFT descriptors. Chem. Phys. Lett. 671, 138-141. doi: 10.1016/j.cplett.2017.01.038

Frau, J., Muñoz, F., and Glossman-Mitnik, D. (2016a). A molecular electron density theory study of the chemical reactivity of cis- and trans-resveratrol. Molecules 21:1650. doi: 10.3390/molecules21121650

Frau, J., Muñoz, F., and Glossman-Mitnik, D. (2016b). A theoretical study of the chemical reactivity of neohesperidin dihydrochalcone through conceptual DFT descriptors. SDRP J. Comput. Chem. Mol. Model. 1:3. doi: 10.25177/JCCMM.1.2.3

Frau, J., Muñoz, F., and Glossman-Mitnik, D. (2016c). Validation of the Koopmans' theorem by means of the calculation of the conceptual DFT descriptors of three fluorescent DNA staining dyes. Chem. Inform. 2:7. doi:10.21767/24706937.100021

Frau, J., Muñoz, F., and Glossman-Mitnik, D. (2017b). A comparison of the Minnesota family of density functionals for the calculation of conceptual DFT descriptors: citrus flavonoids as a test case. Res. J. Chem. Sci. 7, 46-58.

Frau, J., Muñoz, F., and Glossman-Mitnik, D. (2017c). A conceptual DFT study of the chemical reactivity of magnesium octaethylprphyrin (MgOEP) as predicted by the Minnesota family of density functionals. Química Nova 40, 402-406. doi: 10.21577/0100-4042.20170004

Frau, J., Muñoz, F., and Glossman-Mitnik, D. (2017d). Application of DFT concepts to the study of the chemical reactivity of some resveratrol derivatives through the assessment of the validity of the "Koopmans in DFT" (KID) procedure. J. Theor. Comput. Chem. 16:1750006. doi: $10.1142 /$ S0219633617500067 
Frau, J., Ramis, R., and Glossman-Mitnik, D. (2017e). Computational prediction of the preferred glycation sites of model helical peptides derived from Human Serum Albumin (HSA) and Lysozyme Helix 4 (LH4). Theor. Chem. Acc. 136:39. doi: 10.1007/s00214-017-2070-6

Frisch, M. J., Trucks, G. W., Schlegel, H. B., Scuseria, G. E., Robb, M. A., Cheeseman, J. R., et al. (2018). Gaussian 09 Revision D.01. Wallingford, CT: Gaussian Inc.

Gázquez, J., Cedillo, A., and Vela, A. (2007). Electrodonating and electroaccepting powers. J. Phys. Chem. A 111, 1966-1970. doi: 10.1021/jp065459f

Gledhill, J. D., De Proft, F., and Tozer, D. J. (2016). Range-separation parameter in tuned exchange-correlation functionals: successive ionizations and the Fukui function. J. Chem. Theory Comput. 12, 4879-4884. doi: 10.1021/acs.jctc.6b00709

Glossman-Mitnik, D. (2013a). A comparison of the chemical reactivity of naringenin calculated with M06 family of density functionals. Chem. Cent. J. 7, 155-161. doi: 10.1186/1752-153X-7-155

Glossman-Mitnik, D. (2013b). Computational nanochemistry study of the chemical reactivity properties of the rhodamine B molecule. Proc. Comput. Sci. 18, 816-825. doi: 10.1016/j.procs.2013.05.246

Glossman-Mitnik, D. (2014a). Chemical reactivity theory within DFT applied to the study of the prunin flavonoid. Eur. Int. J. Sci. Techn. 3, 195-207.

Glossman-Mitnik, D. (2014b). Computational chemistry of natural products: a comparison of the chemical reactivity of isonaringin calculated with the M06 family of density functionals. J. Mol. Model. 20:2316. doi: $10.1007 /$ s00894-014-2316-3

Glossman-Mitnik, D. (2014c). “Computational nanochemistry report of the molecular structure, spectra and chemical reactivity properties of pheophorbide A," in Design and Applications of Nanomaterials for Sensors, ed J. M. Seminario (Dordrecht: Springer Science + Business Media), 359-402.

Glossman-Mitnik, D. (2014d). "Computational nanochemistry study of the molecular structure, spectra and chemical reactivity properties of the BFPF green fluorescent protein chromophore," in Biosensors Nanotechnology, eds A. Tiwari and A. P. Turner (Hoboken, NJ: John Wiley \& Sons), 201-238.

Halgren, T. A. (1996a). Merck molecular force field. I. Basis, form, scope, parameterization, and performance of MMFF94. J. Comput. Chem. 17, 490519.

Halgren, T. A. (1996b). Merck molecular force field. II. MMFF94 van der Waals and electrostatic parameters for intermolecular interactions. J. Comput. Chem. $17,520-552$.

Halgren, T. A. (1996c). Merck molecular force Field. V. Extension of MMFF94 using experimental data, additional computational data, and empirical rules. J. Comput. Chem. 17, 616-641.

Halgren, T. A. (1999). MMFF VI. MMFF94s option for energy minimization studies. J. Comput. Chem. 20, 720-729.

Halgren, T. A., and Nachbar, R. B. (1996). Merck molecular force field. IV. Conformational energies and geometries for MMFF94. J. Comput. Chem. 17, 587-615.

Henderson, T. M., Izmaylov, A. F., Scalmani, G., and Scuseria, G. E. (2009). Can short-range hybrids describe long-range-dependent properties? J. Chem. Phys. 131:044108. doi: 10.1063/1.3185673

Hofmann, T. (1998). 4-Alkylidene-2-imino-5-[4-alkylidene-5-oxo-1,3-imidazol2-inyl]aza- methylidene-1,3-imidazolidine: a novel colored substructure in melanoidins formed by maillard reactions of bound Arginine with glyoxal and furan-2-carboxaldehyde. J. Agric. Food Chem. 46, 3896-3901.

Jacquemin, D., Moore, B., Planchat, A., Adamo, C., and Autschbach, J. (2014). Performance of an optimally tuned range-separated hybrid functional for 00 electronic excitation energies. J. Chem. Theory Comput. 10, 1677-1685. doi: $10.1021 / \mathrm{ct} 5000617$

Karolewski, A., Kronik, L., and Kümmel, S. (2013). Using optimally tuned range separated hybrid functionals in ground-state calculations: consequences and caveats. J. Chem. Phys. 138:204115. doi: 10.1063/1.4807325

Karolewski, A., Stein, T., Baer, R., and Kümmel, S. (2011). Communication: tailoring the optical gap in light-harvesting molecules. J. Chem. Phys. 134, 151101-151105. doi: 10.1063/1.3581788

Koppen, J. V., Hapka, M., Szczeniak, M. M., and Chalasinski, G. (2012). Optical absorption spectra of gold clusters $\mathrm{Au}(\mathrm{n})(\mathrm{n}=4,6,8,12,20)$ from longrange corrected functionals with optimal tuning. J. Chem. Phys. 137:114302. doi: $10.1063 / 1.4752433$
Kronik, L., Stein, T., Refaely-Abramson, S., and Baer, R. (2012). Excitation gaps of finite-sized systems from optimally tuned range-separated hybrid functionals. J. Chem. Theory Comput. 8, 1515-1531. doi: 10.1021/ct2009363

Kuritz, N., Stein, T., Baer, R., and Kronik, L. (2011). Charge-transfer-like $\pi \rightarrow \pi^{*}$ excitations in time-dependent density functional theory: a conundrum and its solution. J. Chem. Theory Comput. 7, 2408-2415. doi: 10.1021/ct2002804

Lima, I. T., Prado, A. d. S., Martins, J. B. L., de Oliveira Neto, P. H., Ceschin, A. M., da Cunha, W. F., et al. (2016). Improving the description of the optical properties of carotenoids by tuning the long-range corrected functionals. $J$. Phys. Chem. A 120, 4944-4950. doi: 10.1021/acs.jpca.5b12570

Manna, A. K., Lee, M. H., McMahon, K. L., and Dunietz, B. D. (2015). Calculating high energy charge transfer states using optimally tuned range-separated hybrid functionals. J. Chem. Theory Comput. 11, 1110-1117. doi: 10.1021/ct501018n

Marenich, A., Cramer, C., and Truhlar, D. (2009). Universal solvation model based on solute electron density and a continuum model of the solvent defined by the bulk dielectric constant and atomic surface tensions. J. Phys. Chem. B 113, 6378-6396. doi: 10.1021/jp810292n

Martínez-Araya, J. I., and Glossman-Mitnik, D. (2015). The substituent effect from the perspective of local hyper-softness. An example applied on normeloxicam, meloxicam and 4-meloxicam: non-steroidal anti-inflammatory drugs. Chem. Phys. Lett. 618, 162-167. doi: 10.1016/j.cplett.2014.11.004

Martínez-Araya, J. I., Grand, A., and Glossman-Mitnik, D. (2015). Towards the rationalization of catalytic activity values by means of local hyper-softness on the catalytic site: a criticism about the use of net electric charges. Phys. Chem. Chem. Phys. 17, 29764-29775. doi: 10.1039/c5cp03822g

Martínez-Araya, J. I., Salgado-Morán, G., and Glossman-Mitnik, D. (2013a). Computational nanochemistry report on the oxicams - conceptual DFT and chemical reactivity. J. Phys. Chem. B 117, 6639-6651. doi: 10.1021/jp400241q

Martínez-Araya, J. I., Salgado-Morán, G., and Glossman-Mitnik, D. (2013b). Computational nutraceutics: chemical reactivity properties of the flavonoid naringin by means of conceptual DFT. J. Chem. 2013:8. doi: $10.1155 / 2013 / 850297$

Mendoza-Huízar, L. H., Salgado-Morán, G., Ramírez-Tagle, R., and GlossmanMitnik, D. (2016). A theoretical quantum study of the intramolecular interactions and chemical reactivity of polymorphs $\mathrm{A}$ and $\mathrm{B}$ of famotidine in the gas, DMSO, and aqueous phases. Comput. Theor. Chem. 1075, 54-62. doi: 10.1016/j.comptc.2015.11.007

Moore, B. II, and Autschbach, J. (2013). Longest-wavelength electronic excitations of linear cyanines: the role of electron delocalization and of approximations in time-dependent density functional theory. J. Chem. Theory Comput. 9, 4991-5003. doi: 10.1021/ct400649r

Morell, C., Grand, A., and Toro-Labbé, A. (2005). New dual descriptor for chemical reactivity. J. Phys. Chem. A 109, 205-212. doi: 10.1021/jp046577a

Morell, C., Grand, A., and Toro-Labbé, A. (2006). Theoretical support for using the $\Delta f(\mathbf{r})$ descriptor. Chem. Phys. Lett. 425, 342-346. doi: $10.1016 /$ j.cplett.2006.05.003

Niskanen, M., and Hukka, T. I. (2014). Modeling of photoactive conjugated donoracceptor copolymers: the effect of the exact HF exchange in DFT functionals on geometries and gap energies of oligomer and periodic models. Phys. Chem. Chem. Phys. 16, 13294-13305. doi: 10.1039/C4CP01165A

Parr, R., Szentpaly, L., and Liu, S. (1999). Electrophilicity index. J. Am. Chem. Soc. 121, 1922-1924.

Parr, R., and Yang, W. (1984). Density functional approach to the frontier-electron theory of chemical reactivity. J. Am. Chem. Soc. 106, 4049-4050.

Pereira, T. L., Leal, L. A., da Cunha, W. F., Timóteo de Sousa Júnior, R., Ribeiro Junior, L. A., et al. (2017). Optimally tuned functionals improving the description of optical and electronic properties of the phthalocyanine molecule. J. Mol. Model. 23:71. doi: 10.1007/s00894-017-3246-7

Peverati, R., and Truhlar, D. G. (2011). Improving the accuracy of hybrid metaGGA density functionals by range separation. J. Phys. Chem. Lett. 2, 2810-2817. doi: $10.1021 /$ jz201170d

Peverati, R., and Truhlar, D. G. (2012). Screened-exchange density functionals with broad accuracy for chemistry and solid-state physics. Phys. Chem. Chem. Phys. 14, 16187-16191. doi: 10.1039/C2CP42576A

Phillips, H., Geva, E., and Dunietz, B. D. (2012a). Calculating off-site excitations in symmetric donor-acceptor systems via time-dependent density functional theory with range-separated density functionals. J. Chem. Theory Comput. 8, 2661-2668. doi: 10.1021/ct300318g 
Phillips, H., Zheng, S., Hyla, A., Laine, R., Goodson, T. III, Geva, E., et al. (2012b). Ab initio calculation of the electronic absorption of functionalized octahedral silsesquioxanes via time-dependent density functional theory with range-separated hybrid functionals. J. Phys. Chem. A 116, 1137-1145. doi: 10.1021/jp208316t

Refaely-Abramson, S., Baer, R., and Kronik, L. (2011). Fundamental and excitation gaps in molecules of relevance for organic photovoltaics from an optimally tuned range-separated hybrid functional. Phys. Rev. B 84:075144-8. doi: $10.1103 /$ PhysRevB.84.075144

Rizzi, G. P. (1997). Chemical structure of colored maillard reaction products. Food Rev. Int. 13, 1-28.

Salgado-Morán, G., Ruiz-Nieto, S., Gerli-Candia, L., Flores-Holguín, N., FavilaPérez, A., and Glossman-Mitnik, D. (2013). Computational nanochemistry study of the molecular structure and properties of ethambutol. J. Mol. Model. 19, 3507-3515. doi: 10.1007/s00894-013-1884-y

Sastre, S., Frau, J., and Glossman-Mitnik, D. (2017). Computational prediction of the protonation sites of Ac-Lys-(Ala)n-Lys-NH2 peptides through conceptual DFT and MEDT descriptors. Molecules 22:458. doi: 10.3390/molecules 22030458

Soto-Rojo, R., Baldenebro-López, J., and Glossman-Mitnik, D. (2015). Study of chemical reactivity in relation to experimental parameters of efficiency in coumarin derivatives for dye sensitized solar cells using DFT. Phys. Chem. Chem. Phys. 17, 14122-14129. doi: 10.1039/C5CP 01387A

Stein, T., Kronik, L., and Baer, R. (2009a). Prediction of charge-transfer excitations in coumarin-based dyes using a range-separated functional tuned from first principles. J. Chem. Phys. 131:244119. doi: 10.1063/1. 3269029
Stein, T., Kronik, L., and Baer, R. (2009b). Reliable prediction of charge transfer excitations in molecular complexes using time-dependent density functional theory. J. Am. Chem. Soc. 131, 2818-2820. doi: 10.1021/ja8087482

Sun, H., and Autschbach, J. (2014). Electronic energy gaps for $\pi$-conjugated oligomers and polymers calculated with density functional theory. J. Chem. Theory Comput. 10, 1035-1047. doi: 10.1021/ct4009975

Weigend, F. (2006). Accurate coulomb-fitting basis sets for H to R. Phys. Chem. Chem. Phys. 8, 1057-1065. doi: 10.1039/b515623h

Weigend, F., and Ahlrichs, R. (2005). Balanced basis sets of split valence, triple zeta valence and quadruple zeta valence quality for $\mathrm{H}$ to $\mathrm{Rn}$ : design and assessment of accuracy. Phys. Chem. Chem. Phys. 7, 3297-3305. doi: 10.1039/B508541A

Yanai, T., Tew, D. P., and Handy, N. C. (2004). A new hybrid exchange-correlation functional using the coulomb-attenuating method (CAM-B3LYP). Chem. Phys. Lett. 393, 51-57. doi: 10.1016/j.cplett.2004.06.011

Zhurko, G., and Zhurko, D. (2012). Chemcraft Program Revision 1.6. Grigoriy A. Zhurko.

Conflict of Interest Statement: The authors declare that the research was conducted in the absence of any commercial or financial relationships that could be construed as a potential conflict of interest.

Copyright (C) 2018 Frau and Glossman-Mitnik. This is an open-access article distributed under the terms of the Creative Commons Attribution License (CC $B Y)$. The use, distribution or reproduction in other forums is permitted, provided the original author(s) and the copyright owner are credited and that the original publication in this journal is cited, in accordance with accepted academic practice. No use, distribution or reproduction is permitted which does not comply with these terms. 